\title{
Comparison between Web Engineering Methods to Develop Multi Web Applications
}

\author{
Karzan Wakil $1,2,{ }^{*}$, Dayang N. A. Jawawi ${ }^{1}$ \\ ${ }^{1}$ University Technology Malaysia-Malaysia. \\ 2 University of Human Development-Iraq. \\ * Corresponding author. Email: karzanwkil@gmail.com \\ Manuscript submitted June 28, 2017; accepted August 4, 2017. \\ doi: $10.17706 /$ jsw.12.10.783-793
}

\begin{abstract}
Web applications act as a source of strength for business growth over the internet and have become the backbone of the e-commerce. With the evolution of these type of applications, numerous features have been added to overcome the issues of complexities of the processes development web engineering methods. These methods are intended for specific purposes, and there has been some lack of the development of web applications, especially concerning the new types of web applications. The problem is that web engineering methods cannot support new features of web applications, furthermore no single method to support all types of web applications. In an attempt to solve this problem, a significant amount of work has been undertaken to improve or extend these methods to develop new types of web applications. In this paper, we make a comparison between web engineering methods (including IFML) to support the features of modern web applications. The result of our comparison will show the capability of the methods to support modern and multi web applications, and help the developers in the process development web applications.
\end{abstract}

Key words: Web engineering methods, web applications, IFML, comparison study.

\section{Introduction}

With the swift web evolution during the period past 1990, web engineering methods were implemented to create web applications. Numerous methods, like Hypermedia Design Model (HDM) [1], as well as Object-Oriented Hypermedia Design Method (OOHDM) [2], facilitated novel approaches, notations plus models initially handling "hypermedia" systems generally, and eventually highlighting web-based systems precisely. The growth of this research line, Web engineering, is examined in Several comparative studies and surveys [3]-[10]. Regardless of a range of uncertainties put forth concerning the need for specialized Web development approaches contrary to "conventional" or "traditional" methods [11].

Web engineering methods for progressing web applications, tend to engage a range of notations and suggest some development procedures, hence employing a standard metamodel as based approaches for the Web domain. Equally enough, the metamodel is the best delineation of the modeling models. The association between metamodels and their aspects, with aptly-formedness guidelines, continues requirements for designing a semantic web. Web engineering methods founded on this metamodels as part of the design facilitated by the metamodels. The common metamodels ought to be a union of the modeling design of renowned web engineering methods allowing for their excellent evaluation and assimilation [12]. Founded on evolution aspects and intricacy, there exist diverse classifications for webs and webs application.

In their study, Aghaei et al. [13] categorized four Web generations founded on the growth of web design and 
includes web 4.0, web 3.0, web 2.0, and web 1.0. A presentation and comparison of these web generations' features have equally signified achieved. It was evident that as of 1989, information space or the Web has had tremendous development and it is adapting towards Artificial Intelligent methods and is seen as a large Web of sophisticated, intelligent communications shortly.

The primary problem facing businesses is that single web engineering methods cannot support all web applications, since each method has been designed for a special aspect. Moreover, a designer needs specific information about approaches to develop web applications. In this paper, we make a comparison between web engineering methods to develop multi web applications. Comparison study best way to find weaknesses and strengths of the methods in the process development web applications, also comparison explains the similarity and differences between methods.

This paper is organized as follows: Section 2 demonstrates related works. Section 3 explains modern web applications. Section 4 explains MDWE methods by which to develop multi web applications. In Section 5, we make a comparison between existing web engineering methods used to develop multi web applications. The last section consists of the conclusion of the comparison and suggestions for future research.

\section{Background}

Within the study area of web methods, there exists comparatively limited literature on such web methods [12], [14]-[17]. While acting as a basis for process improvement and improved product dominance, a vast level of literature in Web engineering has centered on the proposal of tools and methodologies. In their study, Lee et al. [14] assessed the measurements of some particular aspects of Web approaches, such as stages, predominant modeling approaches, navigation source, documentation, techniques to identify user's preference and semantic quality. Nonetheless, the outcomes authenticity may have suffered ambiguity as a result of subjectivity by the researchers hence causing demarcation in the study outcomes. For instance, Koch [8] compares the phases ran through by some given web approaches by engaging a differentiation technique that obscured some key aspects. There exists an apparent deviation in the infiltration where an approach describes a stage. For example, some web approaches tend to propose some textual approaches for various growth phases with others facilitating support tools for the identical phases.

Nonetheless, Montero et al. [16] design a requirement configuration, with the intent of examining web engineering methods for system's hypermedia. Some conditions are obtained from the areas of software engineering as well as web engineering. These conditions are employed in the configuration of methodologies to assess their benefits and demerits. To exemplify the elements of web applications, as well as where the present methods fail in conforming to these conditions, Gu et al. [15] assesses the prerequisites of web approaches. In some key areas, present limitations appear highly significant, such as, the affiliation between operational and information aspects need for support within its dynamic modeling. This study by Gu et al. can be regarded being less biased and vague compared to that by Lee et al, Koch and Montero et al., regardless of the meager methods offered while some extensions were not taken into consideration. Furthermore, Wakil and Jawawi made a comparison between UWE, OOH and WebML through the metamodels and elements [9]. Furthermore, they perform a new comparison between UWE and WebML [8] to show weaknesses and strengths of the methods, these comparisons showed the ability of methods to develop web applications, but not highlighted the type of applications.

Baresi and Morasca [17] carried on three empirical studies focusing the effort needed on designing web applications. The empirical studies were based on the use of W2000, but the hypotheses and results may apply to other methods. By differentiating between web engineering methods in previous works, there were drawbacks and strengths. But certain investigators endeavored to enhance web engineering techniques and metamodels, however until now is not ideal yet for the development of web application. However a number comparison exists but not enough to show capability the methods, for this reason in this paper, we will try to find the best method among web engineering methods to develop multi web applications including new features from 
modern web applications.

\section{Modern Web Applications}

Based on the continuing evolution of features and complexity, there are different categorizations for webs and web applications. An outline, based on the evolution of the web given by Aghaei et al., classified four generations which are: web 1.0, web 2.0, web 3.0 and web 4.0. The features of the generations are presented and contrasted. It came to the point that, since 1989, information space or the web has undergone tremendous development. It is increasingly adapting artificial intelligent methods and is seen as becoming a huge web of sophisticated intelligent communications in the near future [13].

Web applications have differing degrees of complexity. They may be strictly informational or manage full-size and fully-fledged 24/7 e-commerce applications, based on their progress history and their level of difficulty [18]. Wakil and Jawawi [19] decided that the latest web applications are ubiquitous web applications, semantic web applications and intelligent web applications. In addition, there is the Rich Internet Application (RIA) that focuses on the client and server user interface as highlighted in Fig. 3.

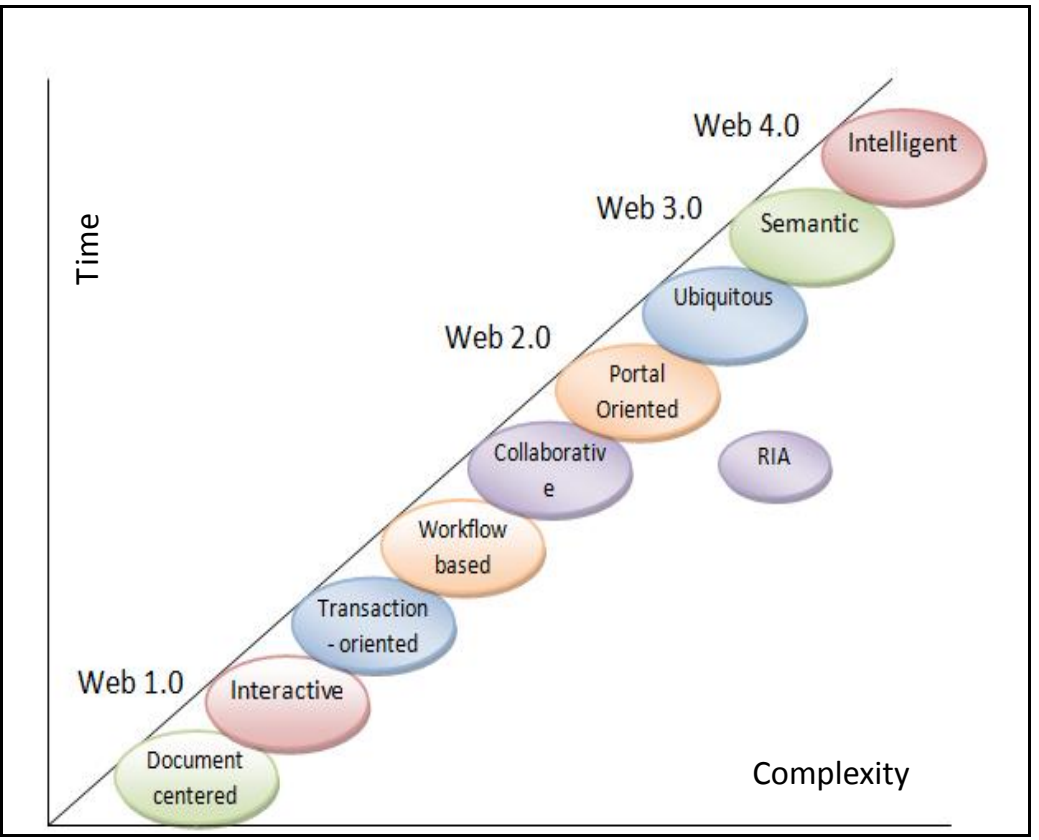

Fig. 1. Web applications complexity history with respect to web evolution[19].

After web 2.0 until web 4.0, the progress of new web applications is based on the outcome above. The latest and most popular web applications centered on complexity and history are: RIA, UWA, SWA, and IWA. Table 1 shows the features of web applications that need to be supported by web engineering methods.

Table 1. Features of Newest web Applications [19]

\begin{tabular}{ll}
\hline \hline Features & Web applications \\
\hline Client/Server, Rich UI, Rich Client & RIA \\
Anywhere, Anytime, Anyplace, Anymedia, Anydevice, Adaptation, Context- aware & UWA \\
Ontology, Rich UI, RDF, Semantic hyperlink, behavior & SWA \\
Web Personalization, Web Mining, Semantic Web, Intelligent Agents & IWA \\
\hline
\end{tabular}

The client/server feature is a feature that helps the software working on the client and server as the same interface through the rich UI. Furthermore, Rich client is a new feature that users mostly utilize through new smart phones, ipads and new electronic devices. The features from UWA possess extraordinary characteristics to present web applications on any device, anywhere or via any media, ensuring high adaptability for the web 
software.

A new feature from semantic web is ontology. The semantic web is mostly used by developers for web site development, especially in Google, and other search engines. This feature is very important to enable users to search easily in order to find information by using semantic hyperlinks. The future feature of web applications from IWA is that of web mining. It is expected that this will help web users to utilize web applications in a smart way.

\section{Web Engineering Methods for Developing Web Applications}

There are a number of web engineering methods which have been proposed for developing web applications. These are shown as follows:

\subsection{Web Modeling Language (WebML)}

Web Modeling Language (WebML) [20] is normally a model-driven method for the design and creation of data-intensive application of webs via waterfall software procedure. The method entails seven phases whereby the software developer conceptualizes diverse web application qualities. This approach is founded on three models [19] which include presentation model, Data model as well as Enhanced Hypertext Model. Within the last 10 years, there has been an improvement of the inventive WebML methodology by engaging primitives in creating diverse web applications aspects as well as fresh application types [21]. Different scholars have pioneered an enhancement of this approach in handling the creation of 'Rich Internet Applications'([22]. Bozzon et al. [23], Toffetti [24] and Fraternali et al. [25]). Precisely, the researchers developed an enhanced such Data model to promote the depiction of temporary and distributed data objects, as well as the Stretch Text model, inclusive of novel primitives to handle the new model for delineating the dynamic aspect of the elements of an RIA user interface, that is, the RIA dynamic model, identical to UML activity diagrams. This method is sustained by a CASE tool identified as WebRatio [26].. In their study, Brambilla and Facca [27]exemplified the key features and requirements of 'semantic web portals' hence proposing an enhanced WebML in tackling the creation of these applications type. While creating social web applications, Fraternali et al. [28] described and examined a set of software models utilized in prevailing community-based Web applications. In addition, the researchers introduced a collection of models illustrative of the way of implementing them by engaging WebRatio and WebML.

\subsection{UML-Based Web Engineering (UWE)}

As initially proposed by Koch and Kraus [29], [30], UML-based Web Engineering (UWE) entails a model-driven development method for Web applications founded on the utility of UML models. Exclusively, the approach engages a UML profile for the specification of the use conditions plus the design qualities. UWE creation method is facilitated by a five-model design plus the transition among them: Presentation model, Navigation model, Process model, Content model as well as the Requirements model. Additionally, ArgoUWE is a CASE tool [31] highly facilitates the UWE by instigating the UML pattern as well as the model transformations. Over the last 10 years, UWE has been employed in Web applications as far as business setting is concerned to customize Rich Internet Applications as well as business Web applications, founded on the use of BPMN (Business Process Modeling Notation) standard. There exist two UWE diversifications in creating Web applications: one suggested by the inventive researchers while the second proposed by some modern researchers.

In their study, Koch et al. [32] devised a method founded on pattern in creating Rich Internet Applications by using UWE. The researchers invented the description of UWE models' extension points for integrating references to RIA development. These patterns are described as UML state models and are incorporated into UW models by using model-to-model transitions or to the eventual Web application code through model-to-text transitions. The other method, suggested by Machado et al. [33], , enhanced the UWE UML profile integrating the aspects needed in modeling the definite RIA aspects. The resulting methodological extension was identified $s$ UWE-R. The authors offered a description of the RIA design inclusive of novel aspects in the process, navigation and 
presentation models, inherent of the design and behavior of UWE profile elements.

\subsection{RUX Method}

Rich User eXperience (RUX) method [34]-[36] is another model-compelled approach centered on the creation of rich user interfaces, that is, the user-client RIA modules. For the purpose of coming up with the eventual user interface, this method suggests a 3-phase method compelled by the design of three models: the Abstract, Concrete and Final interfaces. The RUX-method is incapable of facilitating the design and creation of RIA server modules as it is centered on RIA client modules and user interface. For the creation of the RIA server, the researchers at first suggested reusing the WebML Data and Hypertext models [37]. So as to minimize the RUX-WebML interdependence and confirm the method flexibility, the researchers equally suggested another adjustment for the UWE models engagement [36]. RUX-Tool CASE tool highly facilitates the RUX method [38].

In their study, Linaje et al. [39] described the modifications and conditions required for the integration of semantic annotations within AJAX user interfaces by utilizing the universal Web Consortium WAI-ARIA proposal [40], which highlights the convenience of Web user interface through ontology collection. The suggested solution is integrated, and therefore enhanced, courtesy of EditSAW tool, typically a CASE tool for the creation of accessible Websites by engaging its personal causal approach. In another methodology, Linaje et al. [41] offered a solution by using onto RUX,, that is, an enhancement of the WAI-ARIA ontology

\subsection{Object Oriented Hypermedia Design Method (OOHDM)}

The Object Oriented Hypermedia Design Method (OOHDM) [42] was equally enhanced to handle the establishment of RIA [43]. The suggested method enhances the OOHDM interface model to highlight the creation of the rich user interfaces by engaging ADVs (Abstract Data Views), which delineates all the structural aspects of the rich interface besides being hierarchically presented. As a measure of delineating the interface behavior, the researchers considered the use of ADV-charts, typically some state tools facilitating expressing interface transformations caused by user interactions.

\subsection{Object Oriented Web Solutions (OOWS)}

The object oriented web solutions (OOWS) methodology [44] is a model-compelled method centered on the development Web (2.0) applications and founded on 00-Approach. Another proposal was offered by Valverde and Pastor [45] in their research to address creation of Rich Interfaces, by suggesting an enhancement of this method. In this study, the researchers offered a novel model, that is, the model of Interaction, to identify the RUI components as well as their dynamic behavior. They equally delineated a set of typical interaction models capable of taking place between the users and the RIA interface. An inclusive categorization of all the prevailing methods and a concise explanation of all the methods can be established in the assessment conducted by Toffetti et al. [46].

\subsection{Semantic Hypermedia Design Method (SHDM)}

Semantic Hypermedia Design Method (SHDM) [47] is an ontology-compelled design approach for creating Web applications on Semantic Web. From a 3-ontology set, that is, navigation (describing the application configuration plus the navigational paths), domain (delineating the application data) as well as presentation (stipulating the Websites' conceptualization) the approach creates all the web application modules. In later studies [48-49], researchers enhanced SHDM with a set of primitives in designing RIAs. This enhancement augmented the total presentation ontology elements increased the Web creator functionalities. In this final adaptation, the researchers managed to prevail over the shortage of annotation in their initial Web applications by using RDFa [50].It is important to argue that the inventive SHDM proposal focuses at creating textual annotations on browser-focused RIAs, founded on JavaScript and HTML. This clearly restricts the forms of technology capable of being employed in the RIAs creation. Of late, de Souza and Schwabe (2011) have revised the methodology so as to create web applications capable of functioning with data from Data Web, which is, 
achieving the Lincked data standards. This method is executed using synth proposal, which enhances the design and creation of Data Web applications.

\subsection{Hera Methodology}

HERA [51] is a method used in the configuration of SWISs (semantic web information systems), described as WIS (Web information) that utilize semantic web methods. In particular, the method employs ontology in the depiction of the application's domain knowledge, facilitating the reuse of the prevailing ontology. Nonetheless, the researchers introduce a method for having the generated websites annotated. Using the Hera point of view, the SWIS structural design can be categorized into 3 classes: Semantic, Application and Presentation Layers. There exist two key events in the procedure described within the Hera methodology: data collection and presentation [52] [53], where data generated from various sources is integrated while the designer creates a hypermedia data presentation of the data acquired from the initial phase, respectively. Additionally, Vander Suijs et al [54] suggested a methodology enhancement, identified as Hera-S, in order to promote the configuration of "navigation-focused Web structures over Semantic Web data." Hera-S facilitates the creation of Semantic Web applications that, engaging the Sesame RDF framework [55] for the organization and storage of RDF triples, permit other applications to openly access and adapt the data utilizing the "Sesame RDF framework" [56].

\subsection{Web Requirements Engineering (WebRE)}

WebRE is an approach concerning the handling of Web prerequisites founded on W2000, Navigational Development Method (NDT), OOHDM, and UWE. The concepts of modeling presented in the present study for Web prerequisites specifications are delineated founded on the correspondences of the assessed methods. They are signified as UML metaclasses and make up our WebRE model. The metaclasses delineate the notions without any knowledge concerning its demonstration. They are categorized into two groups, in adherence to the design of UML metamodel: the WebRE behavior package and WebRE structure. The behavior package entails Web Process, Metaclasses Navigation, Web User, Search and User Transaction as illustrated in figure 2.20 [57].

\subsection{Interaction Flow Modeling Language (IFML)}

IFML [58] endorses the platform-independent account on GUI for applications accessed on installed on systems like laptops, desktop computers, tablets, mobile phones and PDAs. The predominant concentration is on the application's design and behavior as recognized as the end user. The language of modeling equally integrates data references and business logic affecting the experience of the user. This is attained accordingly by having the domain model objects referenced by facilitating the data exhibited in the interface and the deeds to be activated through interface interaction. Wakil and jawawi,2017 [59] added IFML to web engineering lifecycle's map, they explained IFML one of the good methods to cover lifecycle, this work help the researcher to follow IFML to solve other web application issues. Moreover they created a framework for combining IFML with other methods[60], this combine provide more ability to IFML for developing web applications. IFML extended for developing mobile applications by [61]. IFML will be one of the best methods because created after ten year experience of WebML.

\section{Comparison between Web Engineering Methods to Develop Multi Web Applications}

In this section, we make a comparison between the existing web engineering methods used to develop multi-web applications. In this comparison, we use the web application features that are presented in Table 1.

The main features of RIA are Client/Server, rich user interface, Generate browser and Generate Plug-in Oriented rich client. Most web engineering methods originally cannot support RIA features, but after improvement or extension was supported, among them IFML originally supported RIA features. Another type of web applications is Semantic Web, this type more popular and more usable type in developing web applications, many applications focused on Semantic web, the Semantic web features related to ontology such as; design 
ontology, import ontology, generate ontology instance, General semantically annotate UIs, and Generate a service Ontology. As shown in Table 2 some web engineering methods can support semantic web and some of them after improvement supported. WebML, OOHDM, OOWS are best methods to support semantic web applications; however other methods can support or have an ability to extend to support semantic web features.

Another type of web applications is Ubiquitous Web application, this type of application not popular type just defined by some researcher in web engineering methods, but very adaptive type especially in their features (anytime/anywhere/anymedia). WebML and SDHM are methods that can support this type of web applications. the latest modern web application in intelligent web application, this type starting at 2016 and will become base of modern and smart web application in the future, the main features are; Web mining, Intelligent Agent and web personalization, this type not applicable by web engineering methods, so we cannot decide which one is the best method to develop this type of web applications. Based on features we can describe each method can support features of intelligent web applications also can support this type of web application.

Table 2. Comparison between Web Engineering Methods to Develop Multi Web Applications

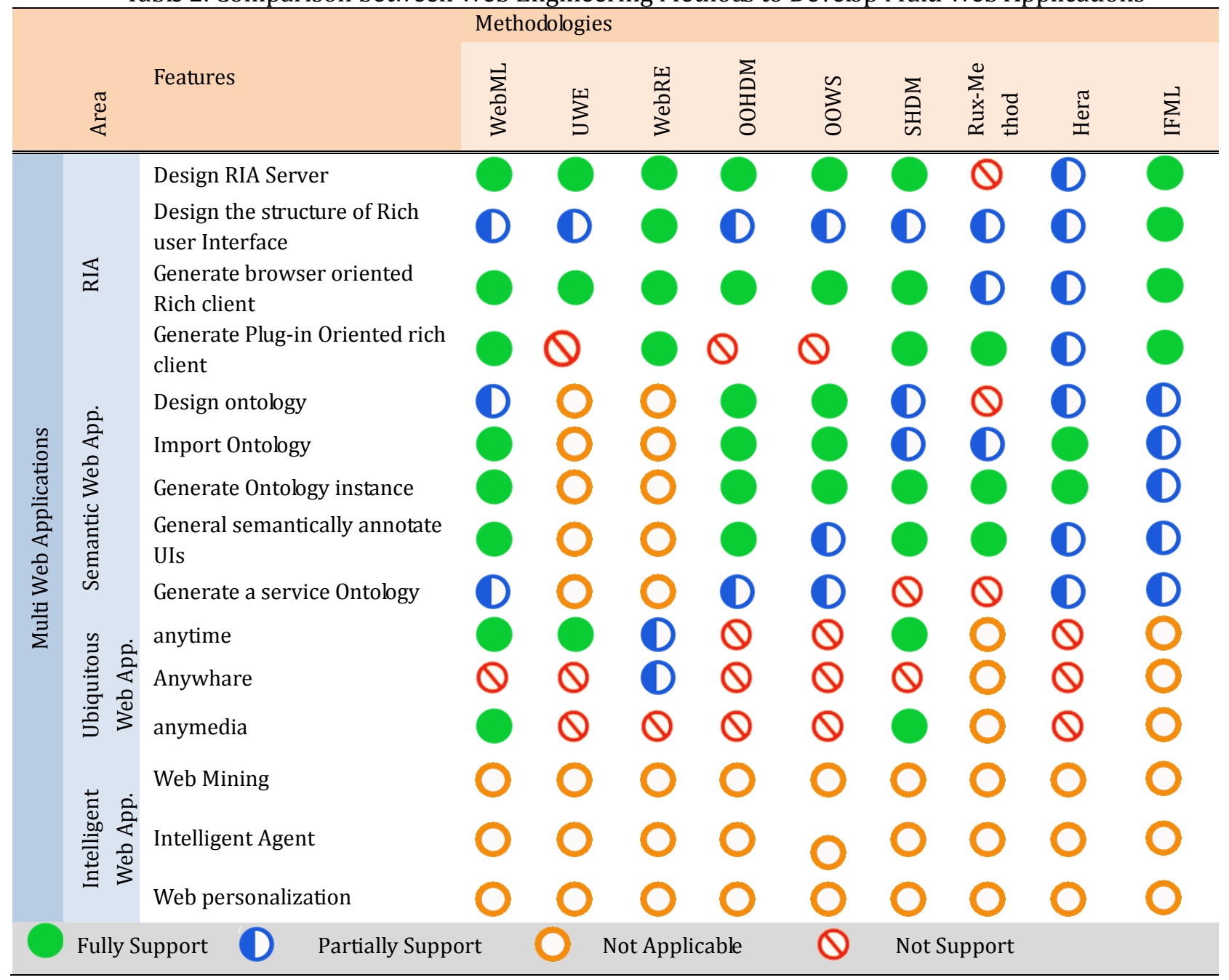

Table 2 above shows a detailed comparison between web engineering methods used for developing multi-web applications. The current methods are already extended to support RIA, especially UWE, WebML, OOHDM, OOWS, and so on. Most methods have been extended to support the semantic web but some of them are not applicable (such as UWE). Even though the methods can support UWA, it still seems that all is not perfect. Moreover, existing web engineering methods do not qualify for intelligent web applications. However, IFML is a new method which supports rich interface RIA but cannot support web applications. However, we can say that it is still the best method for practice. 
Finally, we can conclude about web engineering methods cannot fully support all modern types of web applications, because each method designed for the special task and different concern, so we need to define a new method to support all types of web applications especially modern web applications. however IFML is new but designed after the ten-year experience of WebML and WebRatio, so IFML one of the web engineering methods have an ability to the extension to develop all types of modern web applications.

There are many challenges to extension web engineering methods, for example, the architecture of the method, models, metamodels. The methods have a different type of models and a different number of models; moreover, number and type of elements are different. Another challenge is process development web applications, each method focused on a phase of web engineering life cycle. Another challenge is tool support to design models and metamodels.

\section{Conclusion and Future Work}

In this paper, we have made a comparison between web engineering methods, through their respective capability to support different web applications. This comparison showed that current methods cannot support all types of web applications. However, IFML is still the best method with which to practice. It can support RIA perfectly, but also needs to improve. It is anticipated that this comparison will be able to help designers with choices as to which method in web engineering is the best for the development of web applications. However a number of challenges do not allow enhancing web engineering methods but with using a different mechanism and new technologies we can solve that problem, we recommend for researchers to improve the methods by using new mechanism and new technologies to develop multi web applications through the adaptive concept for models and metamodels.

\section{References}

[1] Garzotto, F., Paolini, P. \& Schwabe, D. (1993). HDM - A model-based approach to hypertext application design. ACM Transactions on Information Systems (TOIS), 11(1), 1-26.

[2] Rossi, G., \& Schwabe, D. (2008). Modeling and implementing web applications with OOHDM. Web Engineering: Modelling and Implementing Web Applications.

[3] Barry, C., \& Lang, M. (2001). A survey of multimedia and web development techniques and methodology usage.

[4] Escalona, M. J., \& Koch, N. (2004). Requirements engineering for web applications-a comparative study. J. Web Eng., 2(3), 193-212.

[5] Escalona, M., et al. (2007). The treatment of navigation in web engineering. Advances in Engineering Software, 38(4), 267-282.

[6] Schwinger, W., et al. (2008). A survey on web modeling approaches for ubiquitous web applications. International Journal of Web Information Systems, 4(3), 234-305.

[7] Wakil, K., \& Jawawi, D. N. (2015). Model driven web engineering: A systematic mapping study. E-Informatica Software Engineering Journal, 9(1), 107-142.

[8] Wakil, K., Jawawi, D. N., \& Safi, A. (2015). A comparison of navigation model between UWE and WebML: Homepage development case study. International Journal of Information and Education Technology, 5(9), 650.

[9] Wakil, K., \& Jawawi, D. N. (2014). Metamodels evaluation of web engineering methodologies to develop web applications. International Journal of Software Engineering and Applications, 5(5), 47.

[10] Jawawi, K., \& W. D. N. A. (2016). Analyzing IFML in Web Development Lifecycle.

[11] Lang, M. (2002). Hypermedia systems development: do we really need new methods. Proceedings of the Informing Science+ IT Education Conference, Cork, Ireland.

[12] De, K. N. P. (2001). Software Engineering for Adaptive Hypermedia Systems-Reference Model, Modeling Techniques and Development Process. 
[13] Aghaei, S., Nematbakhsh, M. A., \& Farsani, H. K. (2012). Evolution of the world wide web: From WEB 1.0 to WEB 4.0. International Journal of Web \& Semantic Technology, 3(1), 1-10.

[14] Lee, H., Lee, C., \& Yoo, C. (1998). A scenario-based object-oriented methodology for developing hypermedia information systems. Proceedings of the Thirty-First Hawaii International Conference on System Sciences.

[15] Gu, A., Lowe, D., \& Henderson-Sellers, B. (2002). Web Modelling Languages: The Gap between Requirements and Current Exemplars.

[16] Montero, S., Díaz, P., \& Aedo, I. (2002). Requirements for hypermedia development methods: A survey of outstanding methods. Advanced Information Systems Engineering.

[17] Baresi, L., \& Morasca, S. (2007). Three empirical studies on estimating the design effort of web applications. ACM Transactions on Software Engineering and Methodology, 16(4), 15.

[18] Kappel, G., et al. (2006). Web Engineering. John Wiley \& Sons.

[19] Karzan, W. D. N. A. J., \& Mohd, A. I. (20150. Analyzing modern web applications to recognize features-based web engineering methods. Proceedings of the 7th International Conference on Internet (ICONI), Malaysia, Kualalmpore.

[20] Ceri, S., Fraternali, P., \& Bongio, A. (2000). Web modeling language (WebML): A modeling language for designing Web sites. Computer Networks, 33(1), 137-157.

[21] Stefano, C., et al. (2002). Designing Data-Intensive Web Applications. Morgan Kaufmann Publishers Inc., San Francisco.

[22] Ceri, S., Brambilla, M., \& Fraternali, P. (2009). The history of webml lessons learned from 10 years of model-driven development of web applications, in conceptual modeling: Foundations and applications. Springer.

[23] Bozzon, A., et al. (2006). Conceptual modeling and code generation for rich internet applications. Proceedings of the 6th international conference on Web engineering.

[24] Carughi, G.T. (2007). Conceptual Modeling and Code Generation of Data-Intensive Rich Internet Applications. $\mathrm{PhD}$ thesis, Politecnico di Milano.

[25] Fraternali, P., et al. (2010). Engineering Rich Internet Applications with A Model-Driven Approach. ACM Transactions on the Web (TWEB), 4(2), 7.

[26] Acerbis, R., et al. (2007). Webratio 5: An eclipse-based case tool for engineering web applications. Web Engineering.

[27] Brambilla, M., \& Facca, F. M. (2007). Building semantic web portals with webML. Web Engineering.

[28] Fraternali, P., et al. (2008). Building community-based web applications with a model-driven approach and design pattern. Handbook of research on Web.

[29] Koch, N., \& Kraus, A. (2002). The expressive power of uml-based web engineering. Second International Workshop on Web-Oriented Software Technology.

[30] Koch, N., \& Kraus, A. (2003). Towards a common metamodel for the development of web applications. Web Engineering.

[31] Knapp, A., Koch, N., \& Zhang, G. (2004). Modeling the structure of web applications with argouwe. Web Engineering.

[32] Koch, N., et al. (2009). Patterns for the Model-based Development of RIAs.

[33] Machado, L., \& Ribeiro, J. (2009). UWE-R: An extension to a web engineering methodology for rich internet applications. WSEAS Transactions on Information Science and Applications, 6(4), 601-610.

[34] Linaje, M., Preciado, J. C., \& Sánchez-Figueroa, F. (2007). A method for model based design of rich internet application interactive user interfaces. Web Engineering.

[35] Linaje, M., Preciado, J. C., \& Sánchez-Figueroa, F. (2007). Engineering rich internet application user interfaces over legacy web models. Internet Computing.

[36] Preciado, J. C., et al. (2008). Designing rich internet applications combining uwe and rux-method. Proceedings of the Eighth International Conference on Web Engineering. 
[37] Brambilla, M., et al. (2008). Business process-based conceptual design of rich internet applications. Proceedings of the Eighth International Conference on Web Engineering.

[38] Linaje, M., et al. (2009). Automatic generation of rias using rux-tool and webratio.

[39] Linaje, M., et al. (2009). Obtaining accessible RIA UIs by combining RUX-Method and SAW. Proceedings of the International Workshop on Automated Specification and Verification of Web Systems.

[40] W3C. Accessible Rich Internet Applications (WAI-ARIA) 1.0. (2014). Retrieved from http://www.w3.org/TR/wai-aria/

[41] Linaje, M., et al. (20110. Providing RIA user interfaces with accessibility properties. Journal of Symbolic Computation, 46(2), 207-217.

[42] Schwabe, D., \& Rossi, G. (1998). An object oriented approach to Web-based applications design.

[43] Urbieta, M., et al. (2007). Designing the interface of rich internet applications. Proceedings of the Conference Web.

[44] Valverde, F. (2010). OOWS 2.0: Un método de Ingeniería Web Dirigido por Modelos para la producción de aplicaciones WEB 2.0, Information Systems and Computation. PhD thesis. Universitat Politècnica de València, Valencia.

[45] Valverde, F., \& Pastor, O. (2008). Applying interaction patterns: Towards a model-driven approach for rich internet applications development. Proceedings of the 7th Int. Wsh. Web-Oriented Software Technologies.

[46] Toffetti, G., et al. (2011). STATE-of-the-art ad tre ds i the systematic developme t of rich i ter et applicatio S. Journal of Web Engineering, 10(1), 070-086.

[47] Lima, F., \& Schwabe, D. (2003). Modeling applications for the semantic web. Web Engineering.

[48] Fialho, A. T., \& Schwabe, D. (2007). Enriching hypermedia application interfaces. Web Engineering.

[49] W3C. Model-based User Interfaces Incubator Group. (2009). Retrieved from http://www.w3.org/2005/Incubator/model-based-\%20ui/wiki/SHDM_-_Semantic_\%20Hypermedia_Desig n_Method

[50] WWW. W3C. Retrieved from: http://www.w3.org/TR/rdfa-syntax/

[51] Houben, G.-J., et al. (2003). Hera: Development of semantic web information systems. Web Engineering.

[52] Vdovjak, R., et al. (2003). Engineering semantic web information systems in Hera. J. Web Eng.

[53] Frasincar, F., Houben, G.-J., \& Barna, P. (2010). Hypermedia presentation generation in Hera. Information Systems, 35(1), 23-55.

[54] Van, D. S. K., et al. (2006). Hera-S: web design using sesame. Proceedings of the 6th International Conference on Web Engineering.

[55] Broekstra, J., Kampman, A., \& Harmelen, F. V. (2002). Sesame: A generic architecture for storing and querying rdf and rdf schema.

[56] Broekstra, J., \& Kampman, A. (2003). SeRQL: A second generation RDF query language. Proceedings of the SWAD-Europe Workshop on Semantic Web Storage and Retrieval.

[57] Escalona, M. J., \& Koch, N. (2007). Metamodeling the requirements of web systems. Web Information Systems and Technologies.

[58] Brambilla, M., \& Fraternali, P. (2014). Interaction flow modeling language: Model-driven UI engineering of web and mobile apps with IFML.

[59] Wakil, K., \& Jawawi, D. N. (2017). Analyzing interaction flow modeling language in web development lifecycle. International Journal of Advanced Computer Science and Applications.

[60] Wakil, K., \& Jawawi, D. Combining web engineering methods to cover lifecycle.

[61] Brambilla, M., Mauri, A., \& Umuhoza, E. (2014). Extending the interaction flow modeling language (ifml) for model driven development of mobile applications front end. Proceedings of the International Conference on Mobile Web and Information Systems. 


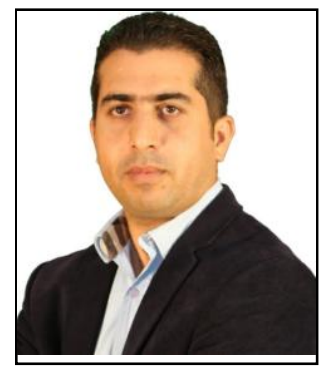

Karzan Wakil is lecturer at the University of Human Development-Iraq and Institute of Training and Educational Development in Sulaimaniyah-Iraq. He received the BSc. degree in computer science from Salahaddin University, Iraq, 2006 and the M.Sc. in computer science from University Technology Malaysia (UTM), Malaysia, 2013. His research areas are web engineering, software engineering and web system..

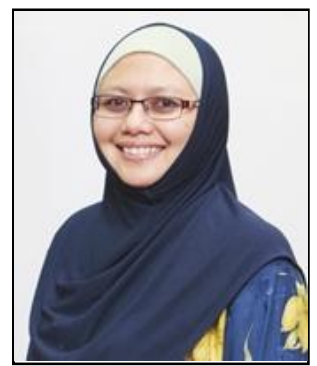

Dayang Norhayati Abang Jawawi is an associate professor in Department of Software Engineering, Faculty of Computing, Universiti Teknologi Malaysia (UTM). She received her B.Sc. in software engineering from Sheffield Hallam University, UK, and her M.Sc. and Ph.D. in the field of software engineering from Universiti Teknologi Malaysia. She has been an academic staff at Software Engineering Department since 1997 and she has served as the head of Department from November 2009 till January 2015. She is a member of the Software Engineering Research Group (SERG), K-Economy Research Alliance, UTM. 